\title{
Implant Dosage Form Category
}

National Cancer Institute

\section{Source}

National Cancer Institute. Implant Dosage Form Category. NCI Thesaurus. Code C154497.

A type of solid pharmaceutical dose form consisting of a mass with a size and shape suitable for implantation, allowing release of active substance(s) over an extended period of time. 